\title{
Optimal Grassmann Manifold Eavesdropping: A Huge Security Disaster for $M-1-2$ Wiretap Channels
}

\author{
Dongyang $\mathrm{Xu}^{* \dagger}$, Pinyi Ren*†, and James A. Ritcey \\ * School of Electronic and Information Engineering, Xi' an Jiaotong University, China \\ ${ }^{\dagger}$ Shaanxi Smart Networks and Ubiquitous Access Research Center, China. \\ ${ }^{\ddagger}$ Department of Electrical Engineering, University of Washington, USA. \\ E-mail: \{xudongyang@stu.xjtu.edu.cn,pyren@mail.xjtu.edu.cn, ritcey@ee.washington.edu \}
}

\begin{abstract}
We in this paper introduce an advanced eavesdropper that aims to paralyze the artificial-noise-aided secure communications. We consider the $M-1-2$ Gaussian MISO wiretap channel, which consists of a $M$-antenna transmitter, a single-antenna receiver, and a two-antenna eavesdropper. This type of eavesdropper, by adopting an optimal Grassmann manifold (OGM) filtering structure, can reduce the maximum achievable secrecy rate (MASR) to be zero by using only two receive antennas, regardless of the number of antennas at the transmitter. Specifically, the eavesdropper exploits linear filters to serially recover the legitimate information symbols and intends to find the optimal filter that minimizes the meansquare error (MSE) in estimating the symbols. During the process, a convex semidefinite programming (SDP) problem with constraints on the filter matrix can be formulated and solved. Interestingly, the resulted optimal filters constitute a complex Grassmann manifold on the matrix space. Based on the filters, a novel expression of MASR is derived and further verified to be zero under the noiseless environment. Besides this, an achievable variable region (AVR) that induces zero MASR is presented analytically in the noisy case. Numerical results are provided to illustrate the huge disaster in the respect of secrecy rate.
\end{abstract}

Index Terms-Physical layer security, eavesdropping, artificial noise, MISO, Grassmann manifold.

\section{INTRODUCTION}

The research on physical layer security has been drawing great attentions with various wireless technologies moving forward. This protection technique, by exploiting the wireless channel variations, can enhance the information secrecy of network which is conventionally protected by the cryptography in the upper layers of network protocol stack. The initial research on the physical layer security refers to the area of information-theoretic secrecy in the wiretap channel which consists of a source, a destination, and an eavesdropper that attempts to intercept the communication between the source and the destination [1]. The authors in [2] investigated a fundamental secrecy measure called secrecy capacity which is defined as the maximum achievable secrecy rate (MASR) between the source and the destination while ensuring that no information can be inferred by the eavesdropper. It was shown that a non-zero secrecy capacity can be achieved between the source and the destination if the channel to the destination is better than that to the eavesdropper. Otherwise, the temporal variations of the channel and the additional degrees of multi-antennas have to be exploited to maintain a positive secrecy rate [3], [4]. The key point is that the perfect knowledge of channel state information (CSI) of the main and eavesdropper channel is required at the source, which however is an unpractical assumption [5].

Therefore, large amounts of research have been concentrated on the secure transmission under imperfect acquisition of eavesdropper's CSI at the source. Among those work, artificial noise (AN), being emitted on top of the informationbearing signal to disrupt the reception at the eavesdropper, gradually comes to be the commonsense of guaranteeing high secrecy rate [6]. With the aid of multi-antenna channels, AN can be placed in the dimensions that cause least interference at the destination but engender dramatic difference between the signal qualities at the destination and the eavesdropper. There exist huge number of AN-based transmission strategies, such as the precoder design and power allocation mechanisms [7]-[10]. In a multiple-input-single-output-multipleantenna eavesdropper (MISOME) system where eavesdroppers are equipped with less antennas than the source, authors in [7] gave an analytical closed-form expression of an achievable secrecy rate and determined an optimal transmit power allocation between the legitimate signal and the AN to maximize the secrecy rate. Authors in [8] studied the AN based scheme in MIMO wiretap channel scenario and presented that the achievable secrecy rate can however be arbitrarily far from the secrecy capacity if the eavesdropper has more antennas than the source and destination. Authors in [9] investigated the optimal physical-layer secure transmission with artificial noise in the wiretap channel and analyzed the impact of the number of transceiver antennas on the secrecy rate. Authors in [10] considered secure downlink transmission in an AN-aided multicell massive MIMO system with a passive multi-antenna eavesdropper. It shows that even with AN assistance, secure transmission can not be guaranteed if the number of eavesdropper antennas is too large. The above AN based schemes assumed that the eavesdropper usually adopt a theoretically capacity-achievable receiver to combat AN. Note that this type of receiver is originally designed without considering the artificial interference. However, there are few literatures discussing the effect of receiver design on the AN based schemes, especially from an eavesdropping perspective. The resulted eavesdropper is therefore underestimated and mistaken to have to eliminate AN interference completely by consuming large antenna resources.

To give a more comprehensive understanding of the functionality of AN mechanism, we in this paper first introduce an optimal Grassmann manifold (OGM) receiver at the eavesdropper which can exploit the statistical property of received 
data to combat AN. We can show that secrecy protection usually achieved by AN can be completely broken down by the eavesdropper with only two receive antennas, no matter how many antennas have been equipped at the source. Our contributions are detailed as follows:

1) Based on the covariance of received data, a whitening operation is performed on the received data to eliminate the power influence of AN. Thereafter, a vectorbased projector is derived so that the eavesdropper can identify the phase information of secrecy information symbols from the whitened data. Finally, an explicit expression of recovered data is presented but subjected to an unknown filtering matrix.

2) Based on the calculation of the mean-squared error (MSE) of estimated symbols, an optimization problem of minimizing the MSE can be formulated in which the filter matrix serves as the optimized variable. Particularly, this problem can be cast as a convex semidefinite program (SDP) and solved with a closed-form optimal solution. Interestingly, the solution includes a series of filters which are verified to lie on a complex Grassmann manifold.

3) Based on the optimal filter structure, the novel expression of MASR is derived. In the noiseless case, the optimal power strategy of achieving MASR is transformed into no power allocation for AN and zero secrecy rate can be always induced. This security disaster is further confirmed through simulations in a wide range of node locations and under a large number of transmit antennas. In the noisy case, an expression of achievable variable region (AVR) is derived and essentially constituted by several factors, such as, a controllable unitary matrix, the eavesdropper's noise level and its distance to the source. To be worse, the eavesdropper can control AVR and then cause zero secrecy rate no matter how many antennas are utilized at the source. We also simulate the AVR and confirm its existence even when the legitimate destination is located in various regions.

The remainder of this paper is structured as follows. Section $\Pi$ presents the system model overview. A novel eavesdropping threat and its technical modeling is given in Section [II] An optimal grassmann eavesdropping manifold is constructed in Section IV. Secrecy performance analysis is performed in Section $\mathrm{V}$ Simulation results are presented in Section VI. Finally, we conclude our work in Section VII.

Notation: Boldface is used for vector a and matrix $\mathbf{A}$. $\mathbf{A}^{H}$ respectively denotes the conjugate transpose of matrix A. $\mathbf{A}(:, n)$ represents the $n$-th column of $\mathbf{A}$. $\|\cdot\|$ denotes the Euclidean norm of a vector or a matrix. $\mathbb{E}\{\cdot\}$ is the expectation operator. $\operatorname{Re}\{\cdot\}$ and $|\cdot|$ respectively represent the real part and the modulus of a complex number.

\section{SYSTEM DESCRIPTION}

We consider a $M-1-2$ wiretap channel where a transmitter Alice with $M$ antennas communicates with a single-antenna legitimate receiver Bob and is also eavesdropped by an eavesdropper Eve with two antennas satisfying $2<M$. We denote the $i$-th transmitted symbol at Alice as $\mathbf{s}_{i} \in$ $C^{M \times 1}, i=1, \ldots, T . T$ represents the total number of symbols (or channel uses) within each coherence interval.

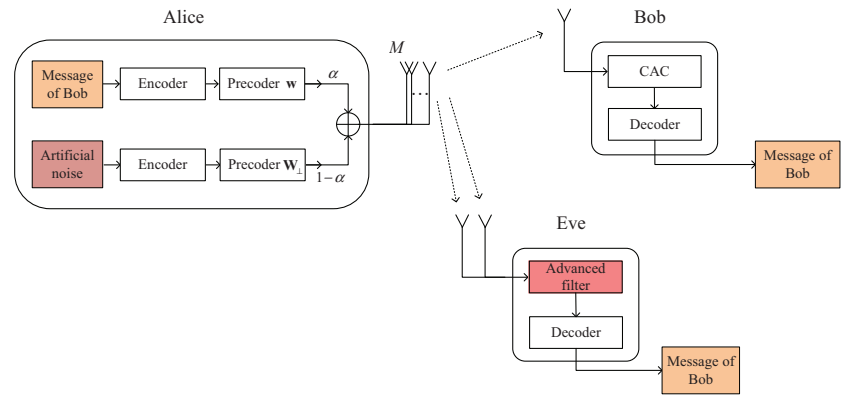

Fig. 1. Illustration of the security threat in artificial-noise-aided $M-1-2$ wiretap channels: An eavesdropper intends to exploit advanced receiver filters to enhance the interception of information belonging to Bob.

The symbols received at Bob and Eve for $i=1, \ldots, T$ are respectively given by:

$$
\begin{aligned}
& \mathbf{y}_{\mathrm{B}, i}=\mathbf{h}_{\mathrm{B}} \mathbf{s}_{i}+\mathbf{n}_{\mathrm{B}, i} \\
& \mathbf{y}_{\mathrm{E}, i}=\mathbf{H}_{\mathrm{E}} \mathbf{s}_{i}+\mathbf{n}_{\mathrm{E}, i}
\end{aligned}
$$

where $\mathbf{h}_{\mathrm{B}} \in \mathbb{C}^{1 \times M}$ denotes the channel from Alice to Bob. $\mathbf{H}_{\mathrm{E}} \in \mathbb{C}^{2 \times M}$ denotes the channel from Alice to Eve. The elements of $\mathbf{h}_{\mathrm{B}}$ and $\mathbf{H}_{\mathrm{E}}$ are assumed to be independent zeromean complex Gaussian random variables with unit variance. The components of $\mathbf{n}_{\mathrm{B}, i}$ and $\mathbf{n}_{\mathrm{E}, i}$ are the equivalent noise with power $\sigma_{\mathrm{B}}^{2}$ and $\sigma_{\mathrm{E}}^{2}$, respectively. We assume $\mathbf{n}_{\mathrm{B}, i}=$ $\beta_{\mathrm{B}}^{-1 / 2} \widetilde{\mathbf{n}}_{\mathrm{B}, i}$ and $\mathbf{n}_{\mathrm{E}, i}=\beta_{\mathrm{E}}^{-1 / 2} \widetilde{\mathbf{n}}_{\mathrm{E}, i}$ where $\beta_{\mathrm{B}}$ and $\beta_{\mathrm{E}}$ are path loss factors respectively from Alice to Bob and Eve. $\widetilde{\mathbf{n}}_{\mathrm{B}, i}$ and $\widetilde{\mathbf{n}}_{\mathrm{E}, i}$ are i.i.d. additive white Gaussian noise (AWGN) samples with variance $\widetilde{\sigma}_{\mathrm{B}}^{2}$ and $\widetilde{\sigma}_{\mathrm{E}}^{2}$, respectively. That's to say, there exists $\sigma_{\mathrm{B}}^{2}=\beta_{\mathrm{E}}^{-1} \widetilde{\sigma}_{\mathrm{B}}^{2}$ and $\sigma_{\mathrm{E}}^{2}=\beta_{\mathrm{E}}^{-1} \widetilde{\sigma}_{\mathrm{E}}^{2}$. Also we have the following assumption:

1) Channels obey the block-fading model where the channel state remains quasi-static over each coherence interval (or channel block), but becomes independent across a different fading block.

2) $T$ within each coherence interval is large enough to allow for invoking random coding arguments [3].

3) $\mathbf{h}_{\mathrm{B}}$ is accurately estimated by Bob and is also known by Alice using a noiseless feedback link from Bob and the knowledge of both $\mathbf{h}_{\mathrm{B}}$ and $\mathbf{H}_{\mathrm{E}}$ is available at Eve [6].

As shown in Fig. 1, the key idea of AN proposed in [6] is that Alice combines the information-bearing symbols intended for Bob with the AN symbols which are generated into the null space of Bob's channel. Thanks to AN, the signal reception of Eve can be disturbed while Bob is not affected. Thus positive secrecy capacity can be achieved and huge secrecy rate gains can be maintained. In this case, the transmitted signal vector $\mathbf{s}_{i}$ at Alice is represented by $\mathbf{s}_{i}=\mathbf{v}_{i}+\mathbf{n}_{i}, i=1, \ldots, T$ where $\mathbf{v}_{i} \in \mathbb{C}^{M \times 1}$ is the transmitted signal vector intended for Bob and $\mathbf{n}_{i} \in \mathbb{C}^{M \times 1}$ is the AN vector. In particular, $\mathbf{v}_{i}$ satisfies $\mathbf{v}_{i}=\mathbf{w} x_{i}$ where $\mathbf{w} \in \mathbb{C}^{M \times 1}$ is the beamforming vector and $x_{i}$ is the the information bearing symbol. AN, independent of $\mathbf{v}_{i}$, satisfies $\mathbf{n}_{i}=\mathbf{W}_{\perp} \mathbf{a}_{i}$ where $\mathbf{W}_{\perp} \in \mathbb{C}^{M \times(M-1)}$ lies in the null space of $\mathbf{H}_{\mathrm{B}}$. It is assumed that $x_{i}$ and elements of $\mathbf{a}_{i}$ are complex Gaussian distributed and independently transmitted in time domain, with variance $\sigma_{x}^{2}$ and $\sigma_{a}^{2}$ respectively. Then the symbols received at Bob and Eve respectively become

$$
\begin{aligned}
& \mathbf{y}_{\mathrm{B}, i}=\mathbf{h}_{\mathrm{B}} \mathbf{w} x_{i}+\mathbf{n}_{\mathrm{B}, i}, i=1, \ldots, T \\
& \mathbf{y}_{\mathrm{E}, i}=\mathbf{H}_{\mathrm{E}} \mathbf{w} x_{i}+\mathbf{H}_{\mathrm{E}} \mathbf{W}_{\perp} \mathbf{a}_{i}+\mathbf{n}_{\mathrm{E}, i}, i=1, \ldots, T
\end{aligned}
$$

We consider that the total power per transmission denoted by $P$ satisfies $P=\sigma_{x}^{2}+(M-1) \sigma_{a}^{2}$. We also denote the 
fraction of total power allocated to the information bearing signal as $\alpha$ and have:

$$
\begin{aligned}
& \sigma_{x}^{2}=\alpha P \\
& \sigma_{a}^{2}=(1-\alpha) P /(M-1), \alpha \in[0,1]
\end{aligned}
$$

\section{Novel EAVESDRopping Threat AND ItS TECHNICAL MODELING}

Based on above AN based context, we in this section introduce a novel statistical eavesdropping threat and give the specific modeling details for this threat, following the analysis of secrecy capacity under traditional receiver assumption for Eve.

\section{A. What We Originally Thought: Eavesdropper Suffers from AN Interference}

To begin with, let us focus on the traditional definition of secrecy capacity which is based on the maximization of mutual information difference. Note that the capacityachievable combiner (CAC) at Eve is not well specified. When AN is introduced for Eve, the same methodology for secrecy capacity is followed since Eve does not know the specific value of $\mathrm{AN}$ and can deem $\mathrm{AN}$ as a colored noise equivalently. In this context, the secrecy capacity and its lower bound can be respectively represented by [6]:

$$
\begin{aligned}
& C_{\mathrm{S}}=\max _{\sigma_{x}^{2}, \sigma_{a}^{2}}\left[C_{\mathrm{B}}-C_{\mathrm{E}}\right]^{+} \\
& C_{\mathrm{S}, \text { lower }}=\max _{\sigma_{x}^{2}, \sigma_{a}^{2}}\left[C_{\mathrm{B}}-C_{\mathrm{E}, \text { upper }}\right]^{+}
\end{aligned}
$$

where $[x]^{+}=\max \{0, x\}$, and

$$
\begin{gathered}
C_{\mathrm{B}}=\log _{2}\left(1+\frac{\sigma_{x}^{2}}{\sigma_{\mathrm{B}}^{2}}\left\|\mathbf{h}_{\mathrm{B}}\right\|^{2}\right), \\
C_{\mathrm{E}}=\log _{2}\left(1+\sigma_{x}^{2} \mathbf{w}^{\mathrm{H}} \mathbf{H}_{\mathrm{E}}^{\mathrm{H}} \mathbf{K}_{\mathrm{E}, 1}^{-1} \mathbf{H}_{\mathrm{E}} \mathbf{w}\right),
\end{gathered}
$$

and

$$
C_{\mathrm{E}, \text { upper }}=\log _{2}\left(1+\frac{M-1}{\alpha^{-1}-1} \mathbf{w}^{\mathrm{H}} \mathbf{H}_{\mathrm{E}}^{\mathrm{H}} \mathbf{K}_{\mathrm{E}, 2}^{-1} \mathbf{H}_{\mathrm{E}} \mathbf{w}\right)
$$

where $\mathbf{w}$ is the beamformer matrix satisfying $\mathbf{w}=\mathbf{h}_{\mathrm{B}} /\left\|\mathbf{h}_{\mathrm{B}}\right\|$. $\mathbf{K}_{\mathrm{E}, 1}=\sigma_{\mathrm{a}}^{2} \mathbf{H}_{\mathrm{E}} \mathbf{W}_{\perp} \mathbf{W}_{\perp}^{\mathrm{H}} \mathbf{H}_{\mathrm{E}}^{\mathrm{H}}+\mathbf{I}_{2}$ is derived under the noisy Eve case while $\mathbf{K}_{\mathrm{E}, 2}=\mathbf{H}_{\mathrm{E}} \mathbf{W}_{\perp} \mathbf{W}_{\perp}^{\mathrm{H}} \mathbf{H}_{\mathrm{E}}^{\mathrm{H}}$ is formulated in the case of a noiseless eavesdropping environment which creates an lower bound on the secrecy capacity [7].

However, the continual utilization of CAC is not feasible for Eve because it can exploit the statistical property of received data, not even necessarily distinguish between signals, and enhance the decoding efficiency of the information symbols that was intercepted.

\section{B. What Eve Can Actually Do: Introducing Statistical Filter to Combat AN Interference}

In this section, we introduce the concept of OGM eavesdropping and show how Eve can exploit the statistical information of received signals to devise the advanced filter. Let us consider the received symbols of length $N(N \leq T)$ in one channel block for Eve:

$$
\mathbf{Y}_{\mathrm{E}}=\mathbf{H}_{\mathrm{E}} \mathbf{w} \mathbf{x}+\mathbf{H}_{\mathrm{E}} \mathbf{W}_{\perp} \mathbf{A}+\mathbf{N}_{\mathrm{E}}
$$

where $\mathbf{Y}_{\mathrm{E}}=\left[\mathbf{y}_{\mathrm{E}, i \in \mathcal{N}}\right] \in \mathbb{C}^{2 \times N}, \mathbf{x}=\left[x_{i \in \mathcal{N}}\right] \in \mathbb{C}^{1 \times N}$, $\mathbf{A}=\left[\mathbf{a}_{i \in \mathcal{N}}\right] \in \mathbb{C}^{(M-1) \times N}, \mathbf{N}_{\mathrm{E}}=\left[\mathbf{n}_{\mathrm{E}, i \in \mathcal{N}}\right] \in \mathbb{C}^{2 \times N} . \mathcal{N}$ rep- resents the time sample set defined as $\mathcal{N} \triangleq\{t \mid 1 \leq t \leq N\}$. We consider a transformation satisfying:

$$
\overline{\mathbf{Y}}_{\mathrm{E}}=\overline{\mathbf{U}} \mathbf{Y}_{\mathrm{E}}
$$

where $\overline{\mathbf{U}} \in \mathbb{C}^{2 \times 2}$ is the unitary matrix and can be known and arbitrarily configured by Eve. We define $\overline{\mathbf{U}}$ as the transform matrix. Then we can rewrite the signal model under $\alpha \in$ $(0,1)$ as follows:

$$
\overline{\mathbf{Y}}_{\mathrm{E}}=\mathbf{H S}+\overline{\mathbf{N}}_{\mathrm{E}}
$$

where $\mathbf{H} \triangleq\left[\begin{array}{ll}\sigma_{x} \overline{\mathbf{U}} \mathbf{H}_{\mathrm{E}} \mathbf{w} & \sigma_{a} \overline{\mathbf{U}} \mathbf{H}_{\mathrm{E}} \mathbf{W}_{\perp}\end{array}\right], \mathbf{S} \triangleq\left[\begin{array}{c}\overline{\mathbf{x}} \\ \overline{\mathbf{A}}\end{array}\right]$ and $\overline{\mathbf{N}}_{\mathrm{E}}=\overline{\mathbf{U}} \mathbf{N}_{\mathrm{E}}$. Each of elements in $\overline{\mathbf{x}}$ and $\overline{\mathbf{A}}$ are respectively power-normalized version of that in $\mathbf{x}$ and $\mathbf{A}$. Based on this model, we give the following steps regarding the novel OGM filter design:

1) Whitening Data: We define the estimation of covariance matrix of $\overline{\mathbf{Y}}_{\mathrm{E}}$ as $\widehat{\mathbf{R}}_{\overline{\mathbf{Y}}_{\mathrm{E}}} \triangleq \overline{\mathbf{Y}}_{\mathrm{E}} \overline{\mathbf{Y}}_{\mathrm{E}}^{\mathrm{H}} / N$ and derive the noiseless version as follows:

$$
\widehat{\mathbf{R}}_{\mathrm{S}}=\widehat{\mathbf{R}}_{\overline{\mathbf{Y}}_{\mathrm{E}}}-\sigma_{\mathrm{E}}^{2} \mathbf{I}_{2}
$$

The whitening operation, defined as $\mathbf{Q} \in \mathbb{C}^{2 \times 2}$, is performed on $\overline{\mathbf{Y}}_{\mathrm{E}}$ to eliminate the second-order statistical information, which is given as follows:

$$
\mathbf{I}_{2}=\mathbf{Q} \widehat{\mathbf{R}}_{\mathrm{S}} \mathbf{Q}^{\mathrm{H}}
$$

After performing singular value decomposition (SVD) on $\widehat{\mathbf{R}}_{\mathrm{S}}$, we have $\widehat{\mathbf{R}}_{\mathrm{S}}=\mathbf{U} \boldsymbol{\Sigma} \mathbf{U}^{\mathrm{H}}$. Based on equation (12), we have:

$$
\mathbf{Q}=\mathbf{X} \boldsymbol{\Sigma}^{-1 / 2} \mathbf{U}^{\mathrm{H}}, \mathbf{X} \mathbf{X}^{\mathrm{H}}=\mathbf{I}_{2}
$$

where $\mathbf{X} \in \mathbb{C}^{2 \times 2}$ is an unknown matrix for further optimization. Then the signal after whitening is given as:

$$
\mathbf{Y}_{\Delta} \triangleq \mathbf{Q Y}=\mathbf{X} \overline{\mathbf{V}} \mathbf{S}+\mathbf{Q} \overline{\mathbf{N}}_{\mathrm{E}}
$$

where $\overline{\mathbf{V}}$ satisfies $\overline{\mathbf{V}}=\boldsymbol{\Sigma}^{-1 / 2}\left[\begin{array}{ll}\boldsymbol{\Sigma}^{1 / 2} & \mathbf{0}\end{array}\right] \mathbf{V}^{\mathrm{H}}$. $\mathbf{V}^{\mathrm{H}}$ is the right eigenmatrix satisfying the matrix SVD with $\mathbf{H}=\mathbf{U}\left[\begin{array}{ll}\boldsymbol{\Sigma}^{1 / 2} & \mathbf{0}\end{array}\right] \mathbf{V}^{\mathrm{H}}$. As we can see, any replacement for the matrix $\mathbf{X}$ with other matrices satisfying equation (14) leaves the covariance of $\mathbf{Y}_{\Delta}$ unchanged, which means that the power influence of AN is removed. The same happens when any replacement for unitary matrices $\mathbf{V}$ with other unitary matrix. However, $\mathbf{X} \overline{\mathbf{V}}^{\mathrm{H}}$ causes a phase error on the symbol estimation of $\mathbf{S}$ at Eve.

2) Projecting the Processed Data onto a Basis: To eliminate the phase error, Eve is designed to project the whitened data onto $\mathbf{X} \overline{\mathbf{V}}^{\mathrm{H}}(:, 1)$ and to estimate the information bearing symbols intended for Bob. We denote the projector as

$$
\mathbf{q}=\mathbf{X} \overline{\mathbf{V}}^{\mathrm{H}}(:, 1)=\mathbf{Q H}(:, 1)
$$

It is obviously that there exists $\|\mathbf{q}\|^{2} \leq 1$. Based on the overall two steps, we can have the following proposition for the symbol-level filtering.

Proposition 1. Given the first column of matrix $\mathbf{H}$, the estimated version of the symbol vector $\overline{\mathrm{x}}$ under the OGM filter with the region $\alpha \in(0,1)$ can be derived as follows:

$$
\widehat{\overline{\mathbf{x}}}=\mathbf{q}^{\mathrm{H}} \mathbf{Q} \overline{\mathbf{Y}}_{\mathrm{E}}
$$

The overall serial filtering process at Eve can be shown in Fig. 2, 


$$
\begin{gathered}
\mathrm{NMSE}=\mathbf{H}(:, 1){ }^{\mathrm{H}} \mathbf{U} \boldsymbol{\Sigma}^{-1 / 2} \mathbf{Z}\left(\mathbf{I}_{2}+\sigma_{\mathrm{E}}^{2} \boldsymbol{\Sigma}^{-1}\right) \mathbf{Z}^{\mathrm{H}} \boldsymbol{\Sigma}^{-1 / 2} \mathbf{U}^{\mathrm{H}} \mathbf{H}(:, 1)-2 \mathbf{H}(:, 1){ }^{\mathrm{H}} \mathbf{U} \boldsymbol{\Sigma}^{-1 / 2} \mathbf{Z}^{\mathrm{H}} \boldsymbol{\Sigma}^{-1 / 2} \mathbf{U}^{\mathrm{H}} \mathbf{H}(:, 1)+1 \\
\mathcal{L}(\mathbf{Z}, \lambda, \mu, \boldsymbol{\Psi})= \\
\quad \operatorname{Tr}\left\{\boldsymbol{\Sigma}^{-1 / 2} \mathbf{U}^{\mathrm{H}} \mathbf{H}(:, 1) \mathbf{H}(:, 1)^{\mathrm{H}} \mathbf{U} \boldsymbol{\Sigma}^{-1 / 2} \mathbf{Z}\left(\mathbf{I}_{2}+\sigma_{\mathrm{E}}^{2} \boldsymbol{\Sigma}^{-1}\right) \mathbf{Z}^{\mathrm{H}}-2 \boldsymbol{\Sigma}^{-1 / 2} \mathbf{U}^{\mathrm{H}} \mathbf{H}(:, 1) \mathbf{H}(:, 1)^{\mathrm{H}} \mathbf{U} \boldsymbol{\Sigma}^{-1 / 2} \mathbf{Z}^{\mathrm{H}}\right\} \\
\quad-\lambda\left\{\operatorname{Tr}\left\{\boldsymbol{\Sigma}^{-1 / 2} \mathbf{U}^{\mathrm{H}} \mathbf{H}(:, 1) \mathbf{H}(:, 1)^{\mathrm{H}} \mathbf{U} \boldsymbol{\Sigma}^{-1 / 2} \mathbf{Z}^{\mathrm{H}}\right\}-1\right\}-\mu\left\{\operatorname{Tr}\left\{\mathbf{Z}^{\mathrm{H}}\right\}-2\right\}-\operatorname{Tr}\left\{\boldsymbol{\Psi} \mathbf{Z}^{\mathrm{H}}\right\} \\
\lambda^{*}=\frac{1-\mathbf{H}(:, 1)^{\mathrm{H}} \mathbf{U} \boldsymbol{\Sigma}^{-1 / 2} \Gamma\left(\boldsymbol{\Sigma}^{-1 / 2} \mathbf{U}^{\mathrm{H}} \mathbf{H}(:, 1) \mathbf{H}(:, 1)^{\mathrm{H}} \mathbf{U} \boldsymbol{\Sigma}^{-1 / 2}\right)\left[\mathbf{I}_{2}+\sigma_{\mathrm{E}}^{2} \boldsymbol{\Sigma}^{-1}\right]^{-1} \boldsymbol{\Sigma}^{-1 / 2} \mathbf{U}^{\mathrm{H}} \mathbf{H}(:, 1)}{\mathbf{H}(:, 1)^{\mathrm{H}} \mathbf{U} \boldsymbol{\Sigma}^{-1 / 2}\left[\mathbf{I}_{2}+\sigma_{\mathrm{E}}^{2} \boldsymbol{\Sigma}^{-1}\right]^{-1} \boldsymbol{\Sigma}^{-1 / 2} \mathbf{U}^{\mathrm{H}} \mathbf{H}(:, 1)}-2
\end{gathered}
$$

\section{CONSTRUCTION OF Optimal GRASSMANN EAVESDROPPING MANIFOLD}

In this section, we formulate an optimization problem of minimizing MSE on the variable matrix $\mathbf{X}$ and derive an optimal Grassmann manifold by solving this problem. Not that we only consider the case where the power ratio satisfies $0<\alpha<1$ in the following. If AN does not exist $(\alpha=1)$, the conventional CAC receiver can be utilized at Eve. The detection of whether AN happens can be based on the rank detection of the covariance matrix $\widehat{\mathbf{R}}_{\mathrm{S}}$. We do not specify the details of detection but focus on the following filter design.

\section{A. Optimization Formulation}

Consider the MSE in estimating symbols $\widehat{\overline{\mathbf{x}}}$ and give the symbol estimation error as follows:

$$
\Delta \overline{\mathbf{x}} \triangleq \widehat{\overline{\mathbf{x}}}-\overline{\mathbf{x}}=\mathbf{q}^{\mathrm{H}} \mathbf{Q} \overline{\mathbf{Y}}_{\mathrm{E}}-\mathbf{S}(1,:)
$$

with the NMSE defined as:

$$
\mathrm{NMSE} \triangleq \mathbb{E}\left\{\|\Delta \overline{\mathbf{x}}\|^{2} / N\right\}
$$

After simplification, the NMSE can be derived as equation (19) if we define $\mathbf{Z}=\mathbf{X}^{\mathrm{H}} \mathbf{X}$. The optimization problem of minimizing NMSE is built up as follows:

$$
\begin{array}{cl}
\min _{\mathbf{Z}} & \operatorname{Tr}\left\{\mathbf{T Z}\left(\mathbf{I}_{2}+\sigma_{\mathrm{E}}^{2} \boldsymbol{\Sigma}^{-1}\right) \mathbf{Z}^{\mathrm{H}}-2 \mathbf{T} \mathbf{Z}^{\mathrm{H}}\right\} \\
\text { s.t. } & \mathbf{T}=\boldsymbol{\Sigma}^{-1 / 2} \mathbf{U}^{\mathrm{H}} \mathbf{H}(:, 1) \mathbf{H}(:, 1)^{\mathrm{H}} \mathbf{U} \boldsymbol{\Sigma}^{-1 / 2} \\
& \mathbf{H}(:, 1)^{\mathrm{H}} \mathbf{U} \boldsymbol{\Sigma}^{-1 / 2} \mathbf{Z}^{\mathrm{H}} \boldsymbol{\Sigma}^{-1 / 2} \mathbf{U}^{\mathrm{H}} \mathbf{H}(:, 1)-1 \leq 0, \\
& \operatorname{Tr}\{\mathbf{Z}\}=2, \\
& \mathbf{Z} \succeq \mathbf{0} .
\end{array}
$$

In this optimization, the second constraint is constructed according to the principle of $\|\mathbf{q}\|^{2} \leq 1$. The third and fourth constraint mean that matrix $\mathbf{Z}$ should satisfy the same rank requirements as $\mathbf{X X}^{\mathrm{H}}$.

\section{B. Optimal Solution}

We can easily prove that the above optimization is a convex problem. Besides, the set of constraint functions is convex and the Slater condition is satisfied. Therefore, the KKT conditions are sufficient and necessary for the optimum solution. Then we build up the Lagrangian as the equation (21) and the KKT conditions for the optimization problem

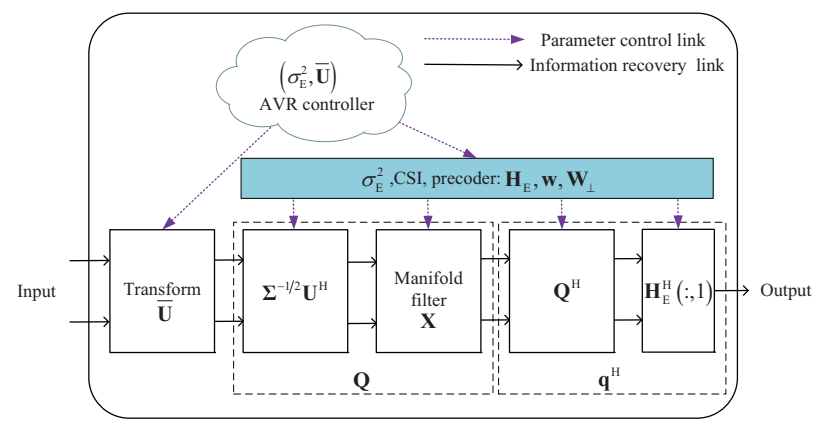

Fig. 2. Architecture of the proposed OGM filter at Eve.

are given by:

$$
\begin{aligned}
\boldsymbol{\Sigma}^{-1 / 2} \mathbf{U}^{\mathrm{H}} \mathbf{H}(:, 1) \mathbf{H}(:, 1){ }^{\mathrm{H}} \mathbf{U} \boldsymbol{\Sigma}^{-1 / 2} \mathbf{Z}\left(\mathbf{I}_{2}+\sigma_{\mathrm{E}}^{2} \boldsymbol{\Sigma}^{-1}\right) \\
-(\lambda+2) \boldsymbol{\Sigma}^{-1 / 2} \mathbf{U}^{\mathrm{H}} \mathbf{H}(:, 1) \mathbf{H}(:, 1){ }^{\mathrm{H}} \mathbf{U} \boldsymbol{\Sigma}^{-1 / 2} \\
-\mu \mathbf{I}_{2}-\mathbf{\Psi}=0(22 \mathrm{a}) \\
\lambda\left\{\mathbf{H}(:, 1)^{\mathrm{H}} \mathbf{U} \boldsymbol{\Sigma}^{-1 / 2} \mathbf{Z}^{\mathrm{H}} \boldsymbol{\Sigma}^{-1 / 2} \mathbf{U}^{\mathrm{H}} \mathbf{H}(:, 1)-1\right\}=0(22 \mathrm{~b}) \\
\mathbf{H}(:, 1)^{\mathrm{H}} \mathbf{U} \boldsymbol{\Sigma}^{-1 / 2} \mathbf{Z}^{\mathrm{H}} \boldsymbol{\Sigma}^{-1 / 2} \mathbf{U}^{\mathrm{H}} \mathbf{H}(:, 1)-1 \leq 0(22 \mathrm{c}) \\
\operatorname{Tr}\left\{\mathbf{Z}^{\mathrm{H}}\right\}-2=0(22 \mathrm{~d}) \\
\operatorname{Tr}\left\{\mathbf{\Psi} \mathbf{Z}^{\mathrm{H}}\right\}=0(22 \mathrm{e}) \\
\mathbf{Z} \succeq \mathbf{0}(22 \mathrm{f}) \\
\mathbf{\Psi} \succeq \mathbf{0}, \lambda \geq 0, \mu \geq 0(22 \mathrm{~g})
\end{aligned}
$$

According to the equation (22d) and (22e), the solution $\Psi^{*}$ should be equal to be zero matrix. Considering the rank constraints on both sides of the equation (22a), we can obtain the solution $\mu^{*}=0$. Then we can get the closed form of $\lambda^{*}$ in equation (23) and then derive the solution $\mathbf{Z}^{*}$ as follows:

$$
\mathbf{Z}^{*}=\left\{\Gamma+\left(\lambda^{*}+2\right) \otimes \mathbf{I}\right\}\left[\mathbf{I}_{2}+\sigma_{\mathrm{E}}^{2} \boldsymbol{\Sigma}^{-1}\right]^{-1}
$$

where $\boldsymbol{\Gamma}$ is the null space of matrix $\boldsymbol{\Sigma}^{-1 / 2} \mathbf{U}^{\mathrm{H}} \mathbf{H}(:, 1) \mathbf{H}(:, 1)^{\mathrm{H}} \mathbf{U} \boldsymbol{\Sigma}^{-1 / 2}$. Based on the equation (13) and (24), a Grassmann manifold $\mathcal{G}(2,2)$ can be thus constructed as the following:

$$
\mathcal{G}(2,2)=\left\{\mathbf{X} \in \mathbb{C}^{2 \times 2}: \mathbf{X X}^{\mathrm{H}}=\mathbf{I}_{2}, \mathbf{X}^{\mathrm{H}} \mathbf{X}=\mathbf{Z}^{*}\right\}
$$

As a special case where the null space is a zero matrix, we can derive a simplified version of $\mathbf{Z}^{*}$ as follows:

$$
\overline{\mathbf{Z}}^{*}=\frac{\left[\mathbf{I}_{2}+\sigma_{\mathrm{E}}^{2} \boldsymbol{\Sigma}^{-1}\right]^{-1}}{\mathbf{H}(:, 1)^{\mathrm{H}} \mathbf{U} \boldsymbol{\Sigma}^{-1 / 2}\left[\mathbf{I}_{2}+\sigma_{\mathrm{E}}^{2} \boldsymbol{\Sigma}^{-1}\right]^{-1} \boldsymbol{\Sigma}^{-1 / 2} \mathbf{U}^{\mathrm{H}} \mathbf{H}(:, 1)}
$$

\section{Secrecy Performance Analysis}

In this section, we firstly derive the exact expression of SINR in a closed form for Eve under the OGM filter. Then we analyse the MASR under different noise level of Eve and 


$$
\begin{aligned}
& \Psi_{1}=\sigma_{x}^{2} \sigma_{a}^{2} A+\sigma_{x}^{2} \sigma_{\mathrm{E}}^{2} B, \Psi_{2}=\sigma_{x}^{2} \sigma_{a}^{4} C, \Psi_{3}=2 \sigma_{x}^{2} \sigma_{\mathrm{E}}^{4} B+2 \sigma_{x}^{2} \sigma_{a}^{2} \sigma_{\mathrm{E}}^{2} D \\
& A=\left|h_{1}\right|^{2}\left\|\mathbf{h}_{4}\right\|^{2}+\left|h_{2}\right|^{2}\left\|\mathbf{h}_{3}\right\|^{2}-2 \operatorname{Re}\left\{h_{1}^{\mathrm{H}} h_{2} \mathbf{h}_{4}^{\mathrm{H}} \mathbf{h}_{3}\right\}, B=\left|h_{1}\right|^{2}+\left|h_{2}\right|^{2}-2 \operatorname{Re}\left\{h_{1}^{\mathrm{H}} h_{2}\right\}, \\
& C=\left\|\mathbf{h}_{4}\right\|^{4}\left|h_{1}\right|^{2}+\left\|\mathbf{h}_{3}\right\|^{4}\left|h_{2}\right|^{2}+\left(\left|h_{1}\right|^{2}+\left|h_{2}\right|^{2}\right)\left\|\mathbf{h}_{3}\right\|^{2}\left\|\mathbf{h}_{4}\right\|^{2}-2\left(\left\|\mathbf{h}_{3}\right\|^{2}+\left\|\mathbf{h}_{4}\right\|^{2}\right) \operatorname{Re}\left\{h_{1}^{\mathrm{H}} h_{2} \mathbf{h}_{4}^{\mathrm{H}} \mathbf{h}_{3}\right\}, \\
& D=\left|h_{1}\right|^{2}\left\|\mathbf{h}_{4}\right\|^{2}+\left|h_{2}\right|^{2}\left\|\mathbf{h}_{3}\right\|^{2}-2 \operatorname{Re}\left\{h_{1}^{\mathrm{H}} h_{2} \mathbf{h}_{4}^{\mathrm{H}} \mathbf{h}_{3}\right\}+\left(\left|h_{1}\right|^{2}+\left|h_{2}\right|^{2}\right) \operatorname{Re}\left\{\mathbf{h}_{4}^{\mathrm{H}} \mathbf{h}_{3}\right\}-\left(\left\|\mathbf{h}_{3}\right\|^{2}+\left\|\mathbf{h}_{4}\right\|^{2}\right) \operatorname{Re}\left\{h_{1}^{\mathrm{H}} h_{2}\right\} .
\end{aligned}
$$

show how Eve can achieve the eavesdropping disaster: zero MASR.

\section{A. Closed Form of SINR Expression}

Theoretically, we assume the data length is enough large and the covariance matrix $\widehat{\mathbf{R}}_{\mathrm{S}}$ can be precisely approximated as $\mathbf{H H}^{\mathrm{H}}$. By using the OGM filter, the SINR of Eve can be derived as the following:

$$
\operatorname{SINR}_{\mathrm{E}}=\frac{\left[\mathbf{H}(:, 1)^{\mathrm{H}}\left(\mathbf{H} \mathbf{H}^{\mathrm{H}}+\sigma_{\mathrm{E}}^{2} \mathbf{I}_{2}\right)^{-1} \mathbf{H}(:, 1)\right]^{2}}{\sigma_{\mathrm{E}}^{2} \mathbf{H}(:, 1)^{\mathrm{H}}\left(\mathbf{H} \mathbf{H}^{\mathrm{H}}+\sigma_{\mathrm{E}}^{2} \mathbf{I}_{2}\right)^{-2} \mathbf{H}(:, 1)}
$$

For simplicity, we define the following equation:

$$
\begin{aligned}
& h_{1} \triangleq \overline{\mathbf{U}}(1,:) \mathbf{H}_{\mathrm{E}} \mathbf{w}, h_{2} \triangleq \overline{\mathbf{U}}(2,:) \mathbf{H}_{\mathrm{E}} \mathbf{w} \\
& \mathbf{h}_{3} \triangleq \overline{\mathbf{U}}(1,:) \mathbf{H}_{\mathrm{E}} \mathbf{W}_{\perp}, \mathbf{h}_{4} \triangleq \overline{\mathbf{U}}(2,:) \mathbf{H}_{\mathrm{E}} \mathbf{W}_{\perp}
\end{aligned}
$$

Then the exact expression of SINR at Eve can be expressed as:

$$
\operatorname{SINR}_{\mathrm{E}}=\frac{\Psi_{1}^{2}}{\sigma_{\mathrm{E}}^{2}\left(\Psi_{2}+\Psi_{3}\right)}
$$

where $\Psi_{1}, \Psi_{2}$ and $\Psi_{3}$ can be shown in equation (30). Based on this equation, we propose the following theorem:

Theorem 1. For the M-1-2 Gaussian wiretap channel, the $M A S R$ is given by:

$$
\max _{\sigma_{x}^{2}, \sigma_{a}^{2}, \sigma_{\mathrm{B}}^{2}} \min _{\sigma_{\mathrm{E}}^{2}, \overline{\mathbf{U}}} C_{S}
$$

where

$$
C_{S}=\left\{\begin{array}{cc}
C_{S}^{-} & 0<\sigma_{x}^{2}<P \\
C_{S}^{+} & \sigma_{x}^{2}=0 \text { or } \sigma_{x}^{2}=P
\end{array}\right.
$$

with

$$
C_{\mathrm{S}}^{-}=\left[\log _{2}\left(\frac{1+\frac{\sigma_{x}^{2}}{\sigma_{\mathrm{B}}^{2}}\left\|\mathbf{h}_{\mathrm{B}}\right\|^{2}}{1+\mathrm{SINR}_{\mathrm{E}}}\right)\right]^{+}
$$

and

$$
C_{S}^{+}=\left[\log _{2}\left(\frac{1+\frac{\sigma_{x}^{2}}{\sigma_{\mathrm{B}}^{2}}\left\|\mathbf{h}_{\mathrm{B}}\right\|^{2}}{1+\frac{\sigma_{x}^{2}}{\sigma_{\mathrm{E}}^{2}}\left\|\mathbf{H}_{\mathrm{E}} \mathbf{h}_{\mathrm{B}} /\right\| \mathbf{h}_{\mathrm{B}}\|\|^{2}}\right)\right]^{+}
$$

When AN exists or equivalently $0<\alpha<1$, OGM filter can be generated as shown in the above section. In this case, the achievable secrecy rate can be easily expressed as $C_{\mathrm{S}}^{-}$. Otherwise, Eve adopts the conventional CAC receiver and the achievable secrecy rate can be expressed as $C_{\mathrm{S}}^{+}$. Note that the minimization operation is considered since $\sigma_{\mathrm{E}}^{2}, \overline{\mathrm{U}}$ can be optimized by Eve to reduce the secrecy rate. In what follows, we will discuss the effect of equivalent noise levels at Eve on the MASR performance.

\section{B. Noiseless Case}

In this case, the equivalent noise level is arbitrary small. An intuitive interpretation is that Bob or Eve is much closer to Alice [6] and/ or have very low noise level. In this context, we have the following theorem:
Theorem 2. With $\sigma_{\mathrm{E}}^{2} \rightarrow 0$, the MASR is equal to zero.

Proof. let us consider the case where $0<\sigma_{x}^{2}<P$. The SINR of Eve will satisfy :

$$
\frac{\mathrm{SINR}_{\mathrm{E}} \sigma_{\mathrm{E}}^{2}}{\sigma_{x}^{2}} \rightarrow \frac{A^{2}}{C}
$$

The secrecy rate is given by:

$$
C_{\mathrm{S}}^{-}=\left[\log _{2}\left(\frac{1+\frac{\sigma_{x}^{2}}{\sigma_{\mathrm{B}}^{2}}\left\|\mathbf{h}_{\mathrm{B}}\right\|^{2}}{1+\frac{\sigma_{x}^{2}}{\sigma_{\mathrm{E}}^{2}} \frac{A^{2}}{C}}\right)\right]_{0<\sigma_{x}^{2}<P}^{+},
$$

Note that $C_{\mathrm{S}}^{-}$increases monotonically with $\sigma_{x}^{2}$ when $\frac{\left\|\mathbf{h}_{\mathrm{B}}\right\|^{2}}{\sigma_{\mathrm{B}}^{2}}>\frac{A^{2}}{\sigma_{\mathrm{E}}^{2} C}$, and decreases monotonically, otherwise. Therefore, the MASR is achieved at $\sigma_{x}^{2}=P$. At this point, the achievable secrecy rate is transformed into $C_{\mathrm{S}}^{+}$. Obviously, $C_{\mathrm{S}}^{+}$is equal to be zero when $\sigma_{\mathrm{E}}^{2} \rightarrow 0$. The proof is completed.

\section{Noisy Case}

In this situation, the equivalent noise can not be ignored at both Bob and Eve whose noise level and distance to Alice can be arbitrarily managed. Besides, it is noted that the variables, such as $h_{1}, h_{2}, \mathbf{H}_{3}$, and $\mathbf{H}_{4}$, in $C_{\mathrm{S}}^{-}$, depend on Eve's CSI, Alice's beamformer and $\overline{\mathbf{U}}$. As a feasible assumption, Eve is enabled to obtain those available information. Especially, $\sigma_{\mathrm{E}}^{2}$ and $\overline{\mathbf{U}}$ are both controllable by Eve. Therefore, the resulted SINR of Eve can be modified and redesigned by itself. Then, we have the following proposition:

Proposition 2. Given perfect CSI and Alice's beamformer at noisy Eve, there always exist variables $\sigma_{\mathrm{E}}^{2}, \overline{\mathrm{U}}$ such that $C_{\mathrm{S}}^{-}$can be restricted to be zero; Particularly, $C_{\mathrm{S}}^{-}$at high SINR is equal to zero $\forall P \in(0, \infty)$ if the following sufficient condition is satisfied:

$$
\begin{aligned}
& \sigma_{\mathrm{E}}^{2}\left(\frac{B^{2}}{A^{2}}-\frac{2 B}{C}\right) \leq 2\left(\frac{B}{A}-\frac{D}{C}\right), \frac{B}{A}<\frac{D}{C}, \\
& \frac{\sigma_{\mathrm{E}}^{2}}{\sigma_{\mathrm{B}}^{2}}<\frac{\left\|\mathbf{H}_{\mathrm{E}} \mathbf{h}_{\mathrm{B}}^{\mathrm{H}} /\right\| \mathbf{h}_{\mathrm{B}} \|^{2}}{\left\|\mathbf{h}_{\mathrm{B}}\right\|^{2}}
\end{aligned}
$$

whichs make log function to be monotonically increasing with $\sigma_{x}^{2}$, or

$$
\sigma_{\mathrm{E}}^{2}\left(\frac{B^{2}}{A^{2}}-\frac{2 B}{C}\right) \geq 2\left(\frac{B}{A}-\frac{D}{C}\right), \frac{B}{A}>\frac{D}{C}
$$

which makes log function to be monotonically decreasing with $\sigma_{x}^{2}$, or

$$
\frac{A^{2}}{\sigma_{\mathrm{E}}^{2} C} \geq \frac{\left\|\mathbf{h}_{\mathrm{B}}\right\|^{2}}{\sigma_{\mathrm{B}}^{2}}, \frac{D}{C}=\frac{B}{A}
$$

which makes log function to be irrelevant with the transmission power. The values of $A, B, C, D$ can be shown in equation (30). 


$$
\begin{gathered}
\mathbf{H}_{\mathrm{E}}=\left[\begin{array}{cccc}
0.60-0.80 \mathrm{i} & 0.06+0.61 \mathrm{i} & 1.05-0.28 \mathrm{i} & -0.49+0.16 \mathrm{i} \\
-0.36+0.47 \mathrm{i} & -0.42+0.29 \mathrm{i} & 0.09+1.53 \mathrm{i} & 0.10-0.59 \mathrm{i}
\end{array}\right] \\
\mathbf{H}_{\mathrm{L}}=\left[\begin{array}{llll}
-0.71+0.12 \mathrm{i} & 0.41+0.94 \mathrm{i} & 0.20+0.91 \mathrm{i} & -1.44+1.49 \mathrm{i}
\end{array}\right], \overline{\mathbf{U}}^{*}=\left[\begin{array}{cc}
-\sqrt{0.2257} & -\sqrt{0.7743} \\
-\sqrt{0.7743} & \sqrt{0.2257}
\end{array}\right]
\end{gathered}
$$

TABLE I

Simulation PARAMETERS AND VALUeS

\begin{tabular}{cc}
\hline Simulation Parameters & Values \\
\hline Path-loss model & Eq. $(26)$ \\
Small-scale fading model & $\mathcal{C N}\left(0, \mathbf{I}_{M}\right)$ \\
Length $N$ of data & $200($ symbols $)$ \\
Noiseless case: $d_{\mathrm{B}}, d_{\mathrm{E}}$ & {$\left[0,10^{4}\right](\mathrm{m})$} \\
Noisy case: available region of $x, y, z$ & $(-\infty, 0],[0, \infty),[0,4]$ \\
Maximum transmit power $P$ & $35(\mathrm{dBm})$ \\
Noisy case: noise power $\widetilde{\sigma}_{\mathrm{B}}^{2}(10 \mathrm{MHZ}$ bandwidth) & $-102(\mathrm{dBm})$ \\
\hline
\end{tabular}

Proof. Let us focus on the monotonicity of the inner log function in $C_{\mathrm{S}}^{-}$. According to its high-order derivative, we can prove that there exists available variables $\sigma_{\mathrm{E}}^{2}, \overline{\mathrm{U}}$ such that the log function is monotone. However, it is exhausting to obtain a sufficient and necessary condition in the form of an explicit expression of those variables. Particularly in high SINR and $0<\alpha<1$, we transform the original $C_{\mathrm{S}}^{-}$ into $C_{\mathrm{S}}^{-}=\left[\log _{2}\left(\frac{\sigma_{\mathrm{E}}^{2}}{\sigma_{\mathrm{B}}^{2}}\left\|\mathbf{h}_{\mathrm{B}}\right\|^{2} \frac{\sigma_{a}^{4} C-2 \sigma_{a}^{2} \sigma_{\mathrm{E}}^{2} D+2 \sigma_{\mathrm{E}}^{4} B}{\sigma_{a}^{4} A^{2}-2 \sigma_{\mathrm{E}}^{2} A B \sigma_{a}^{2}+\sigma_{\mathrm{E}}^{4} B^{2}}\right)\right]^{+}$. Now we introduce the sufficient condition to achieve zero secrecy rate.

When the log function is controlled by Eve to be monotonically increasing with $\alpha$, the condition (37), (38) has to be satisfied. In this situation, the achievable secrecy rate is gradually increased with the increase of transmission power allocated for information bearing signals and the maximum will be achieved at $\alpha=1$. Thus the condition $\frac{\sigma_{\mathrm{E}}^{2}}{\sigma_{\mathrm{B}}^{2}}<\frac{\left\|\mathbf{H}_{\mathrm{E}} \mathbf{h}_{\mathrm{B}} /\right\| \mathbf{h}_{\mathrm{B}}\|\|^{2}}{\left\|\mathbf{h}_{\mathrm{B}}\right\|^{2}}$ should be further satisfied to make $\left.C_{\mathrm{S}}^{+}\right|_{\alpha=1}$ zero.

Otherwise, in order to make the log function monotonically decrease, we determine that the condition (39) should be satisfied so that the maximum is achieved at $\alpha=0$. Obviously, $\alpha=0$ means that there is no transmission process and naturally indicates that the available secrecy rate is equal to zero.

Finally, if $\log$ function in $0<\alpha<1$ is a constant with the variable $\alpha$, we determine the condition (40) to make $C_{\mathrm{S}}^{-}$ zero. The proof is completed.

Moreover, we define the available variable pair as $\left(\sigma_{\mathrm{E}}^{2}, \overline{\mathbf{U}}\right)$ and collect those pairs that satisfy the above proposition to construct an achievable variable region (AVR) for Eve. Therefore, we have the following theorem.

Theorem 3. In noisy case, there always exists an AVR such that MASR is equal to zero.

It is a natural result from the Proposition 2 since the $C_{\mathrm{S}}^{-}$ can be reduced to be zero when $0<\alpha<1$ and otherwise the $C_{\mathrm{S}}^{+}$can be controlled to be zero by Eve through a suitable configuration of $\sigma_{\mathrm{E}}^{2}$.

\section{Simulation Results}

In this section, we evaluate the secrecy performance of $M-1-2$ wiretap channel model in the context of AN-based transmission mode at Alice. We consider the average MASR

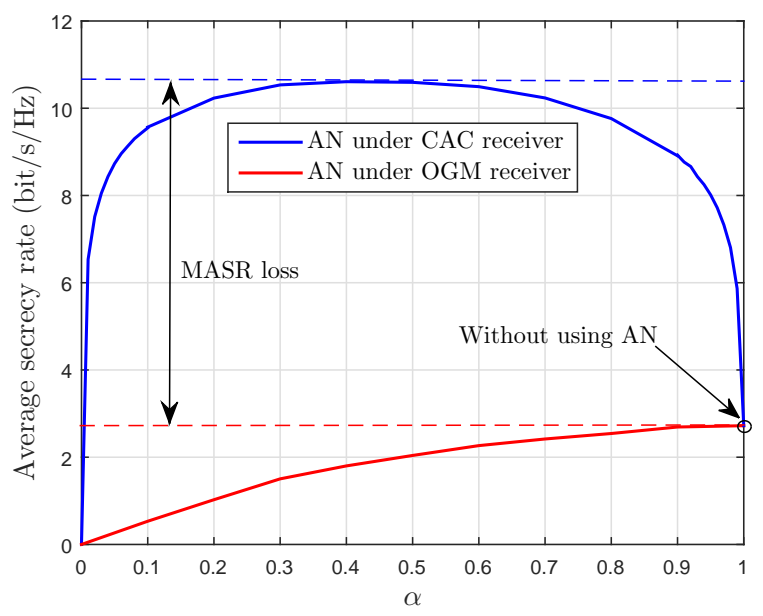

Fig. 3. Average secrecy rate versus the power ratio of $\alpha$ under two types of receivers in the noisy environment.

performance under ergodic scenario and also determine a operation region achieving zero secrecy rate under one channel block. Generally, the path loss expressed in decibels satisfies the following:

$$
P L(d)=P L\left(d_{0}\right)+10 n \log _{10}\left(\frac{d}{d_{0}}\right)
$$

where $d_{0}$ is the reference point at $1 \mathrm{~km}$ and $n$ is known as the path loss exponent. In this paper, we consider a practical 3GPP urban model where $P L\left(d_{0}\right)=148.1$ and $n=3.76$. $\beta_{\mathrm{B}}$ and $\beta_{\mathrm{E}}$ can be derived from $d_{\mathrm{B}}$ and $d_{\mathrm{E}}$ based on equation (41). To better characterize the operation region of noisy Eve, we define several metrics as follows:

$$
x \triangleq \log _{10}\left(\widetilde{\sigma}_{\mathrm{E}}^{2} / \widetilde{\sigma}_{\mathrm{B}}^{2}\right), y \triangleq \log _{10}\left(\beta_{\mathrm{E}} / \beta_{\mathrm{B}}\right), z \triangleq \log _{10}\left(d_{\mathrm{B}}\right)
$$

When Bob is located in different regions $z, x$ and $y$ represent the measures that noisy Eve could take to reduce the secrecy rate to be zero. The detailed simulation parameters can be shown in Table []

Fig. 3 shows the average secrecy rate versus the power ratio of $\alpha$ under different receivers at Eve. In this example, the number of transmit antennas satisfies $M=10$ and the equivalent noise level of Bob is assumed to be equal to that of Eve, that's to say, $\sigma_{\mathrm{B}}^{2}=\sigma_{\mathrm{E}}^{2}$. We also fix the SNR $P / \sigma_{\mathrm{B}}^{2}$ at 30 in $\mathrm{dB}$. As we can see, the average secrecy rate under OGM filter increases with $\alpha$ and achieves the maximum at $\alpha=1$ whereas the MASR under traditional CAC receiver is achieved around at $\alpha=0.5$. Basically, the MASR under OGM receiver is equal to that without using AN at Alice, which means that the AN scheme is completely broken down by Eve. This phenomenon in high SNR is in accord with the result shown in the equation (36). The MASR loss can be as far as $8 \mathrm{bit} / \mathrm{s} / \mathrm{Hz}$.

In Fig. 4, we present the operation region $(x, y, z)$ which is available for noisy Eve to cause zero secrecy rate. Alice adopts the maximum transmission power shown in Table 【 For clarity, we consider one-time channel realization and 


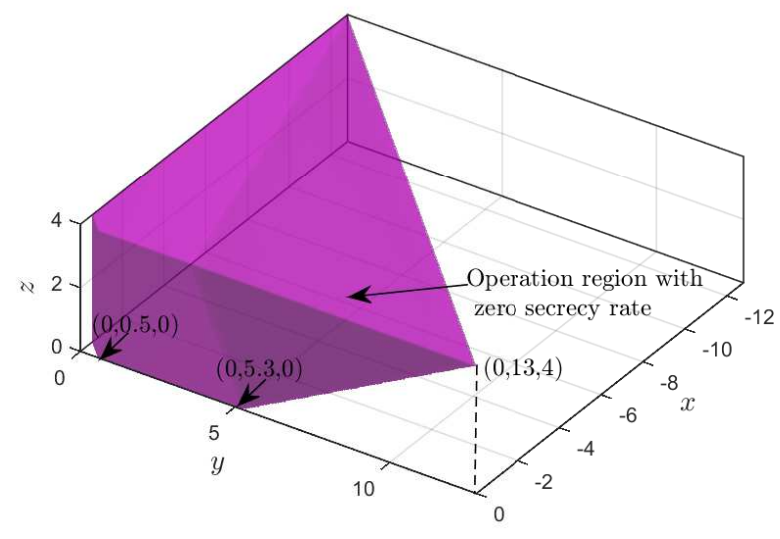

Fig. 4. Operation region $(x, y, z)$ of noisy Eve under the channel model in equation (43) and (44) with the transform matrix $\overline{\mathbf{U}}^{*}$. Our strategy is to achieve the condition (37) and (38).

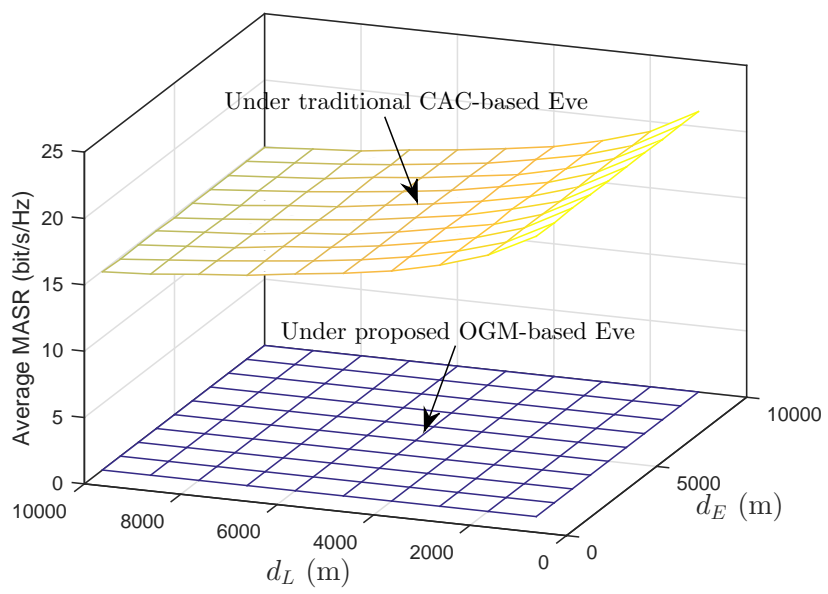

Fig. 5. Average MASR versus the distance $d_{\mathrm{B}}$ and $d_{\mathrm{E}}$ in the noiseless case: $\sigma_{\mathrm{B}}^{2}=\sigma_{\mathrm{E}}^{2} \rightarrow 0$.

focus on arbitrary channel matrices. As an example, the simulated channel matrices and the generated transform matrix are shown in equation (43) and (44). Note that the transform matrix is always available and can be optimized based on the channel matrices and precoders at Eve. As we can see, the operation region is extensive. Especially, OGM based Eve can achieve zero secrecy rate when $z \in[0,4]$ or equivalently $d_{\mathrm{B}} \in\left[1,10^{4}\right]$, by flexibly controlling its path loss and reducing the noise level so that there exist $y \in[0.5,5.3]$ and $x \in[-13,0]$.

Fig.5 demonstrates the average MASR performance versus the distance measure $d_{\mathrm{B}}$ and $d_{\mathrm{E}}$. Alice adopts the maximum transmission power shown in Table [1 The power allocation ratio in traditional $\mathrm{AN}$ based scheme is configured to be 0.5 [7] and the number of antennas is set to be $M=10$. In this case we assume that Bob has same noise level of Eve and the noise level can approach zero. We can see that the secrecy rate, even under the scenario where Bob tries its best to reduce noise, has to be zero and becomes irrelevant to the distances when the OGM-based Eve occurs. Therefore, the traditional expression of secrecy rate under AN-based scheme comes to be imprecise and the CAC receiver assumption for Eve actually causes a huge security for the system.

Fig. 6 shows the average MASR performance under var-

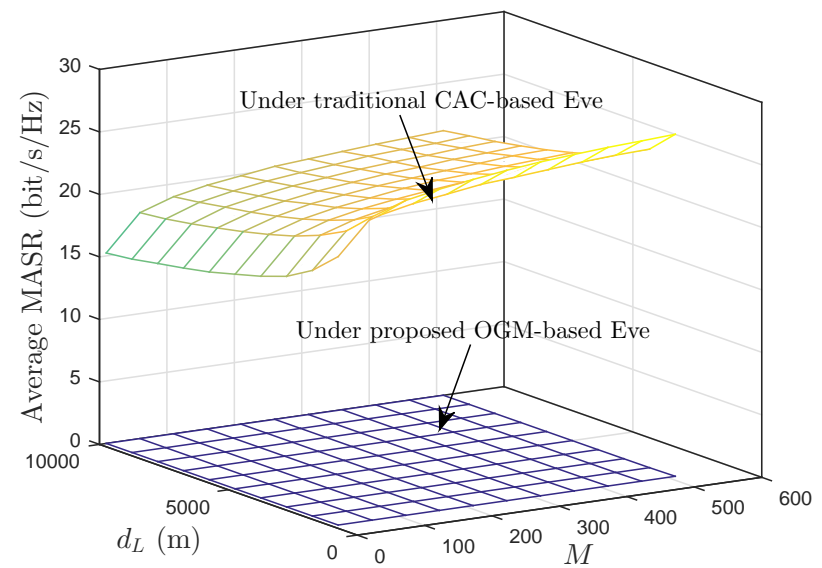

Fig. 6. Average MASR versus the distance $d_{\mathrm{B}}$ and $M$ in the noiseless case: $\sigma_{\mathrm{B}}^{2}=\sigma_{\mathrm{E}}^{2} \rightarrow 0$.

ious $d_{\mathrm{B}}$ and $M$ in the noiseless Eve. Alice adopts the maximum transmission power shown in Table [ In terms of traditional AN based simulations, the location of Eve is fixed at $d_{\mathrm{E}}=1000$ and the power ratio is fixed at $\alpha=0.55$ which is verified to be optimal in [7]. As we can see, the MASR under traditional AN based scheme is gradually enhanced with the increase of $d_{\mathrm{B}}$ and $M$. However, the MASR under OGM-based Eve is always equal to be zero. This result is irrelevant to the variation of $d_{\mathrm{B}}$ and the increase of $M$. This is because the high-SINR level induced by the OGM filter can suppress the high array gains which are brought by Alice for Bob and usually guaranteed by very large number of transmit antennas.

\section{CONCLUSIONS}

In this paper, we introduced a novel eavesdropping behavior which is targeted for AN based secure transmission. We demonstrated how Eve utilized a series of optimal filters to combat the influence of $\mathrm{AN}$ and proved that the maximum achievable secrecy rate which is subjected to this advanced eavesdropper was compelled to be zero in the case of both noiseless and noisy case. Simulation results showed that both the distance measure, either from Alice to Bob or to Eve, actually could not eliminate or reduce this disaster impact. This terrible phenomenon also occurred even though the number of transmit antennas is increased significantly.

\section{REFERENCES}

[1] A. D. Wyner, "The wire-tap channel," Bell Sys. Tech. J., vol. 54, pp. 1355-1387, Oct. 1975.

[2] S. Leung-Yan-Cheong and M. Hellman, "The Gaussian wire-tap channel," IEEE Trans. Inf. Theory, vol. 24, no. 4, pp. 451-456, July, 1978.

[3] Y. Liang, H. V. Poor, and S. Shamai, " Secure communications over fading channels," IEEE Trans. Inf. Theory, vol. 54, no. 6, pp. 24702492, Jun. 2008.

[4] A. Mukherjee, S. A. Fakoorian, J. Huang, and A. L. Swindlehurst, Principles of physical layer security in multiuser wireless networks: A survey," IEEE Commun. Surveys Tuts., vol. 16, no. 3, pp.1550-1573, Mar. 2014.

[5] A. Khisti and G. Wornell, "Secure transmission with multiple antennas I: The MISOME wiretap channel," IEEE Trans. Inf. Theory, vol. 56, no. 7 , pp. 3088-3104, Jul. 2010.

[6] R. Negi and S. Goel, "Guaranteeing secrecy using artificial noise," IEEE Trans. Wireless Commun., vol. 7, no. 6, pp. 2180-2189, Jun. 2008.

[7] X. Zhou and M. R. McKay, "Secure transmission with artificial noise over fading channels: Achievable rate and optimal power allocation," IEEE Trans. Veh. Technol., vol. 59, no. 8, pp. 3831-3842, Oct. 2010. 
[8] A. Khisti and G. W. Wornell, "Secure transmission with multiple antennasłPart II: The MIMOME wireltap channel," IEEE Trans. Inf. Theory, vol. 56, no. 11, pp. 5515-5532, Nov. 2010.

[9] N. Yang, M. Elkashlan, T. Q. Duong, J. Yuan, and R. Malaney, "Optimal transmission with artificial noise in MISOME wiretap channels," IEEE Trans. Veh. Technol., vol. 65, no. 4, pp. 2170-2181, Apr. 2016.

[10] J. Zhu, R. Schober, and V. K. Bhargava, "Secure transmission in multicell massive MIMO systems," IEEE Trans. Wireless Commun., vol. 13, no. 9, pp. 4766-4781, Sep. 2014. 